\title{
COMUNICAÇÃO
}

\section{PREVALENCE OF HUMAN INTESTINAL PARASITES IN THE TOWN OF ALPERCATA, MINAS GERAIS, BRAZIL}

\author{
Odair Genaro
}

There is no previous report about the prevalence of human intestinal parasites in the town of Alpercata, located in the Eastern area of the State of Minas Gerais, Brazil. In order to assess the current status of intestinal parasitic infections, fresh stool specimens were obtained from 1,573 subjects in the time frame between July and August, 1990. The subjects represent about $10 \%$ of the local population estimated in 15,000 habitants. The stool specimens were submmited to the sedimentation technique ${ }^{1}$. Form every specimen three thick films were made, stained with a drop of iodine solution, and immediately examined in the microscope. Table 1 summarizes the results obtained. For most parasites the group more frequently parasitized was that of shoolchildren with ages between 6 and 10. Schistosoma mansoni was the exception, with the highest prevalence occurring between 11 and 30 years of age. The maintenance of soiltransmitted helminthic infections caused by Ascaris lumbricoides, Trichiuris trichiura, Strongyloides stercoralis and hookworm in the town of Alpercata ocurrs most probably in the peridomicile, where the children stay frequently during the day. The relatively high prevalence of Taenia sp is explained by the absence of sanitary control of the meat (pork and cow). Pork highly infected with Cysticercus cellulosae could be found at a local meat shop.

The town of Alpercata is poorly urbanized having basically a rural appearance. Adequate sewage lines are non-existent and so is water treatment which comes basically from homemade wells. These last observations are probably enough to justify the prevalence of Giardia lamblia and Entamoeba histolytica. A survey of the molluscs capable of serving as intermediate hosts for Schistosoma mansoni in water collections of the municipality yielded important observations. A small creek runs through the dowtown area, collecting much of the improvised sewage line. This creek then dumps into a main and secondary ponds serving as play areas for children and adults throughout the year. In the main pond, 292 Biomphalaria glabrata (Planorbidae) varying from 0.2 to $3.7 \mathrm{~cm}$ in diameter and 5 Drepanotrema sp were collected. Examination of the $B$. glabrata revealed a rate of infection by S. mansoni of $12 \%$.

These findings demonstrate the poor health status prevailing in this town which claims attention from the sanitary authorities.

\section{ACKNOWLEDGEMENTS}

I am grateful to the community of Alpercata for active collaboration in these work and the students of the School of Pharmacy at the Universidade Federal de Ouro Preto for their skilled technical assistance. I am also indebted to Dr. A. M. Siqueira for critical reading of the manuscript.

\section{REFERENCE}

1. Hoffman WA, Pons JA, Janer JL. Sedimentation - concentration method in schistosomiasis mansoni. Puerto Rico Journal of Public Health and Tropical Medicine 9:283-291, 1934.

Address to: Dr. Odair Genaro. Departamento de Ciências Biológicas, Universidade Federal de Ouro Preto, 35400 OURO PRETO, MG, Brasil

Recebido para publicação em 06/05/91 
Comunicação. Genaro O. Prevalence of human intestinal parasites in the town of Alpercata, Minas Gerais, Brazil. Revista da Sociedade Brasileira de Medicina Tropical 24:181-182, jul-set, 1991

Table 1 - Prevalence of human intestinal parasites among 1,573 habitants in the town of Alpercata, Minas Gerais, Brazil.

\begin{tabular}{|c|c|c|c|c|c|c|c|c|c|c|c|c|c|c|}
\hline \multirow{2}{*}{ Age } & & \multicolumn{8}{|c|}{ Helminths } & \multicolumn{5}{|c|}{ Protozoans } \\
\hline & & A & $H_{w}$ & Tsp & Sm & Tt & Ev & $\mathrm{Hn}$ & Ss & $\mathrm{Gl}$ & Eh & Ec & En & lb \\
\hline \multirow{2}{*}{$0-1$} & $\mathrm{~F}$ & 2 & 1 & 0 & 0 & 0 & 0 & 0 & 0 & 2 & 0 & 0 & 1 & 0 \\
\hline & $M$ & 6 & 1 & 0 & 0 & 0 & 0 & 0 & 1 & 0 & 1 & 0 & 1 & 0 \\
\hline \multirow{2}{*}{$2-5$} & $F$ & 23 & 2 & 0 & 1 & 5 & 4 & 3 & 1 & 17 & 13 & 9 & 7 & 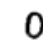 \\
\hline & $M$ & 30 & 5 & 1 & 0 & 6 & 1 & 2 & 1 & 16 & 16 & 23 & 9 & 0 \\
\hline \multirow{2}{*}{$6-10$} & $F$ & 38 & 15 & 2 & 8 & 16 & 3 & 8 & 2 & 23 & 22 & 28 & 34 & 0 \\
\hline & M & 36 & 23 & 3 & 6 & 10 & 2 & 7 & 2 & 22 & 15 & 30 & 14 & 0 \\
\hline \multirow{2}{*}{$11-15$} & $\mathrm{~F}$ & 29 & 12 & 1 & 14 & 6 & 1 & 1 & 1 & 8 & 18 & 17 & 15 & 1 \\
\hline & M & 23 & 22 & 1 & 13 & 9 & 2 & 6 & 1 & 9 & 13 & 25 & 11 & 1 \\
\hline \multirow{2}{*}{$16-20$} & $F$ & 10 & 9 & 0 & 12 & 0 & 3 & 0 & 0 & 3 & 10 & 13 & 9 & 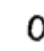 \\
\hline & $M$ & 8 & 14 & 1 & 11 & 2 & 0 & 3 & 1 & 9 & 9 & 14 & 11 & 0 \\
\hline \multirow{2}{*}{$21-30$} & $\mathrm{~F}$ & 15 & 14 & 2 & 9 & 1 & 2 & 1 & 1 & 4 & 16 & 24 & 14 & 0 \\
\hline & $M$ & 5 & 21 & 1 & 20 & 0 & 1 & 1 & 1 & 6 & 10 & 11 & 13 & 0 \\
\hline \multirow{2}{*}{$31-40$} & $F$ & 19 & 18 & 3 & 6 & 2 & 2 & 0 & 0 & 4 & 16 & 25 & 11 & 0 \\
\hline & M & 4 & 15 & 0 & 9 & 0 & 4 & 1 & 0 & 5 & 10 & 10 & 6 & 0 \\
\hline \multirow{2}{*}{$41 \cdot 50$} & $\mathrm{~F}$ & 7 & 5 & 2 & 0 & 2 & 0 & 0 & 1 & 3 & 4 & 8 & 8 & 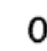 \\
\hline & $M$ & 0 & 11 & 0 & 5 & 0 & 0 & 0 & 1 & 4 & 6 & 7 & 6 & 0 \\
\hline \multirow{2}{*}{$>50$} & $\mathrm{~F}$ & 7 & 9 & 2 & 1 & 0 & 3 & 0 & 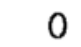 & 3 & 10 & 18 & 8 & 0 \\
\hline & $M$ & 8 & 16 & 1 & 6 & 0 & 1 & 1 & 1 & 1 & 10 & 13 & 8 & 0 \\
\hline \multirow{2}{*}{ Total } & $\mathrm{F}$ & 150 & 85 & 12 & 51 & 32 & 18 & 13 & 0 & 67 & 109 & 142 & 107 & 2 \\
\hline & $M$ & 120 & 128 & 8 & 70 & 27 & 11 & 21 & 8 & 72 & 90 & 133 & 79 & 2 \\
\hline \multicolumn{2}{|l|}{$\begin{array}{l}\text { Both } \\
\text { sexes }\end{array}$} & 270 & 213 & 20 & 121 & 59 & 29 & 34 & 14 & 139 & 199 & 275 & 186 & 4 \\
\hline \multicolumn{2}{|l|}{$\begin{array}{l}\text { Prevalence } \\
\text { rates }\end{array}$} & 17.1 & 13.5 & 1.3 & 7.7 & 3.8 & 1.8 & 2.7 & 0.9 & 8.8 & 12.6 & 17.5 & 11.8 & 0.3 \\
\hline \multicolumn{4}{|c|}{$\begin{array}{l}\mathrm{Al}=\text { Ascaris lumbricoides } \\
\mathrm{Hw}=\text { Hookworm } \\
\mathrm{Tsp}=\text { Taenia } \mathrm{sp} \\
\mathrm{Sm}=\text { Schistosoma mansoni } \\
\mathrm{Tt}=\text { Trichuris trichiura }\end{array}$} & $\begin{array}{l}\mathrm{Ev}= \\
\mathrm{Hn}= \\
\mathrm{Ss}= \\
\mathrm{Gl}=\end{array}$ & $\begin{array}{l}\text { Enterc } \\
\text { Hyme } \\
\text { Stron } \\
\text { Giard }\end{array}$ & $\begin{array}{l}\text { bius } \\
\text { nolep } \\
\text { gyloid }\end{array}$ & $\begin{array}{l}\text { ermic } \\
\text { s nana } \\
\text { es ster }\end{array}$ & lis & & $\begin{array}{l}E \mathrm{~h}= \\
\mathrm{Ec}= \\
E \mathrm{n}= \\
\mathrm{b}=\end{array}$ & $\begin{array}{l}=\text { Entan } \\
=\text { Entan } \\
=\text { Endo } \\
=\text { lodan }\end{array}$ & $\begin{array}{l}\text { moeba } \\
\text { moeba } \\
\text { limax } \\
\text { moeba }\end{array}$ & $\begin{array}{l}\text { histo } \\
\text { a coli } \\
\text { nana }\end{array}$ & \\
\hline
\end{tabular}

